\title{
Solving the problem of constraints due to Dirichlet boundary conditions in the context of the mini element method.
}

\author{
Ouadie Koubaiti ${ }^{1}$, Ahmed Elkhalfi ${ }^{1}$ Jaouad El-mekkaoui ${ }^{2}$, and Nikos Mastorakis ${ }^{3}$,
}

\begin{abstract}
In this work, we propose a new boundary condition called ${ }_{C A ; B}$ to remedy the problems of constraints due to the Dirichlet boundary conditions.

We consider the 2D-linear elasticity equation of Navier-Lamé with the condition $C A ; B$. The latter allows to have a total insertion of the essential boundary condition in the linear system obtained without going through a numerical method like the lagrange multiplier method, this resulted in a non-extended linear system easy to reverse. We have developed the mixed finite element method using the mini element space $(\mathrm{P} 1+$ bubble, $\mathrm{P} 1)$. Finally we have shown the efficiency and the feasibility of the limited condition $C A ; B$.
\end{abstract}

Keywords-Navier Lamé equation, $C A ; B$ generalized condition, mini-element, Matlab, Abaqus.

\section{INTRODUCTION}

The objective of this paper is to present the solution of all the difficulties due to the standard boundary conditions. This is possible by solving the Navier-Lam equation with the generalized boundary condition $C_{A, B}$ using the mixed finite element method $(P 1+$ bubble, $P 1)$. At the same time, we show all the advantages offered by this quality of boundary conditions. In this sense, we calculate the displacement and its divergence simultaneously by the intermediary of another auxiliary unknown called the divergence of displacement. We present two types of comparison: First, we compare the results produced by $(P 1+$ bubble, $P 1)$ and those provided by the Abaqus system. Second, we compute the speed of convergence $\alpha$ obtained by each of the two numerical methods $(P 1+$ bubble, $P 1)$ and the finite element method implemented in the article (J. Alberty et al. 2002, [1]), using linear regression. An analytical example is used to validate the accuracy, convergence and robustness of the present mixed finite element method for elasticity in order to evaluate the efficiency of this method, as well as its usefulness.

Indeed, we will calculate the approximate displacement $u_{h}$ for each of the two methods. They are programmed by Matlab, whose code contains a routine which calculates the errors between the calculated solution and the analytical solution

Ouadie Koubaiti and Ahmed Elkhalfi: Department of Mechanical engineering,Faculty of Sciences and technics, Sidi Mohammed ben abdellah University, Fez, Morocco e-mail: kouba108@gmail.com. Jaouad Elmekkaoui: Department of Mathematics, Faculty Polydisciplinary of BeniMellal, Beni-Mellal, Morocco . Nikos Mastorakis: Hellenic Naval Academy, Greece and Technical university Sofia, Sofia, Bulgaria. E-mail: mastorakis4567@gmail.com presented in the reference (J. Alberty et al. 2002, [1]). When we calculate the solution of the system : $-\mu \Delta u-(\lambda+\mu) \nabla \nabla . u=f$ for a given mesh, we obtain an approximate value of the solution $u_{h}$. Consequently, as the mesh is finer then the solution is more improved.

We consider the following theoretical relation :

$$
\left\|u-u_{h}\right\|_{1, \Omega}=\beta h^{\alpha},
$$

$\beta$ is a positive constant, $h$ is the step of the mesh and $\alpha$ the speed of convergence.

For the calculation of $\left\|u-u_{h}\right\|_{1, \Omega}$, we use the standard $\|$. $\|_{1, \Omega}$ defined below. Knowing that $\left\|u-u_{h}\right\|_{1, \Omega}$ and $h$ the mesh step, we want to calculate $\alpha$ the speed of convergence of the solution. For this, the simplest way to proceed is to compose the logarithm in the equation (1).

We obtain:

$$
\log \left(\left\|u-u_{h}\right\|_{1, \Omega}\right)=\log (\beta)+\alpha \cdot \log (h),
$$

We note that $\log \left(\left\|u-u_{h}\right\|_{1, \Omega}\right)$ is an affine function for the variable $\log (h)$ and $\alpha$ presents the slope.

To find the value of $\alpha$, we compute $\left(\left\|u-u_{h}\right\|_{1, \Omega}\right)$ in different meshes, then we plot the graph on the logarithmic scale of ( $\left.\left\|u-u_{h}\right\|_{1, \Omega}\right)$ according to the log of step $h$. We get the slope of the straight line. In practice, the points are not exactly aligned to obtain the value of $\alpha$. Indeed, we perform a linear regression in the direction of the least squares, that is to say we take for $\alpha$ the slope of the line which approaches all the points.

\section{A. New generalized condition $C_{A, B}$}

We propose the following boundary condition:

$$
C_{A, B}: A u+B\left(\mu \frac{\partial u}{\partial n}+\lambda(\nabla . u) n\right)=g, \text { on } \partial \Omega=\Gamma,
$$

$A$ and $B$ are two invertible and bounded matrix functions belonging to $L^{\infty}(\Gamma)$, with $\Gamma=\Gamma_{D} \cup \Gamma_{N}$. These two matrices are of order 2 for the $2 \mathrm{D}$ case and of order 3 for the $3 \mathrm{D}$ case. We are building these new boundary conditions in order to generalize all types of standard boundary conditions (Dirichlet, Neumann, Robin, ...).

Indeed, we obtain the Dirichlet condition when $\|\mid B\|$ is negligible before ||$|A| \mid$, and on the other hand the condition of Neumann cannot be practically the only boundary condition, so we are talking about the Robin or mixed condition which are well presented by the new boundary condition $C_{A, B}$. 
To illustrate the operation of this boundary condition, we consider the following example, in which a rectangular domain and $\Gamma=\cup_{i=1}^{4} \Gamma_{i}$ its edge: we pose $\Gamma_{D}=\Gamma_{3}$ et $\Gamma_{N}=\Gamma_{1} \cup \Gamma_{2} \cup \Gamma_{4}$, we consider the following boundary conditions :

$$
\left\{\begin{array}{rr}
u=(a(x, y), b(x, y)), & \text { sur } \Gamma_{3} \\
\mu \frac{\partial u}{\partial n}+\lambda \nabla . u n=(c(x, y), d(x, y)), & \text { sur } \Gamma_{1}, \\
\mu \frac{\partial u}{\partial n}+\lambda \nabla . u n=0 & \text { sur } \Gamma_{2}, \\
\mu \frac{\partial u}{\partial n}+\lambda \nabla . u n=0 & \text { sur } \Gamma_{4} .
\end{array}\right.
$$

Assuming that the functions $a, b, c, d$ are non-zero and bounded on $\Gamma$, the system (4) can be expressed in the form of the boundary condition :

$$
C_{A, B}: A u+B\left(\mu \frac{\partial u}{\partial n}+\lambda(\nabla \cdot u) n\right)=g, \text { sur } \partial \Omega=\Gamma .
$$

If we define the displacement $u$ on $\bar{\Omega}$, then the matrix writing of the boundary condition $C_{A, B}$ is written in the form:

$$
\begin{array}{r}
\left(\begin{array}{cc}
\frac{1}{a(x, y)} & 0 \\
0 & \frac{1}{b(x, y)}
\end{array}\right)\left(\begin{array}{l}
u_{1} \mid \Gamma_{\Gamma_{D}} \\
u_{2} \mid \Gamma_{D}
\end{array}\right)+ \\
\left(\begin{array}{cc}
\frac{1}{c(x, y)} & 0 \\
0 & \frac{1}{d(x, y)}
\end{array}\right)\left(\begin{array}{l}
\left.\left.\mu \frac{\partial u_{1}}{\partial n}\right|_{\Gamma_{N}}+\lambda\left(\nabla \cdot u_{\mid \Gamma_{N}}\right) n_{1}\right) \\
\left.\left.\mu \frac{\partial u_{2}}{\partial n}\right|_{\Gamma_{N}}+\lambda\left(\nabla \cdot u_{\mid \Gamma_{N}}\right) n_{2}\right)
\end{array}\right)= \\
\left(\begin{array}{l}
\xi_{1}(x, y)+\xi_{3}(x, y) \\
\xi_{1}(x, y)+\xi_{3}(x, y)
\end{array}\right)
\end{array}
$$

For $i=1$ or 3 , we define the following functions :

$$
\xi_{i}(x, y)=\left\{\begin{array}{r}
1 \text { si }(x, y) \in \Gamma_{i}, \\
0 \text { sinon } .
\end{array}\right.
$$

According to the system (6), just take:

$$
A=\left(\begin{array}{cc}
\frac{1}{a(x, y)} & 0 \\
0 & \frac{1}{b(x, y)}
\end{array}\right), B=\left(\begin{array}{cc}
\frac{1}{c(x, y)} & 0 \\
0 & \frac{1}{d(x, y)}
\end{array}\right)
$$

We set the condition $C_{A, B}$ in the following way :

$$
C_{A, B}: A u+B\left(\mu \frac{\partial u}{\partial n}+\lambda(\nabla . u) n\right)=g \mathbf{1}_{\Gamma_{N}}+h \mathbf{1}_{\Gamma_{D}}
$$

with $g$ is a surface force function and $h$ is a displacement function.

- To model the Dirichlet boundary condition $u=h$ using the generalized condition $C_{A, B}$, just take on $\Gamma_{D}$ :

$$
A=\left(\begin{array}{ll}
1 & 0 \\
0 & 1
\end{array}\right), B=\left(\begin{array}{cc}
10^{-10} & 0 \\
0 & 10^{-10}
\end{array}\right)
$$

- To model the Neumann boundary condition :

$\left(\mu \frac{\partial u}{\partial n}+\lambda(\nabla \cdot u) n\right)=g$ using the generalized condition $C_{A, B}$, just take on $\Gamma_{N}$ :

$$
B=\left(\begin{array}{ll}
1 & 0 \\
0 & 1
\end{array}\right), A=\left(\begin{array}{cc}
10^{-10} & 0 \\
0 & 10^{-10}
\end{array}\right)
$$

\section{AdVAntage OF THE GENERALIZED CONDITION AT THE} WEAK PROBLEM LEVEL

\section{A. Possibility of choosing a less restrictive functional space}

In this section we propose the problem of Navier-Lamé with the condition at the edge $C_{A, B}$ [18].We describe a new auxiliary unknown $\psi$ to be able to apply the mixed finite element methods.

$$
\psi=\nabla \cdot u=\frac{\partial u_{1}}{\partial x}+\frac{\partial u_{2}}{\partial y} .
$$

The Navier-lamé equation becomes:

$$
\left\{\begin{aligned}
-\mu \Delta u-(\lambda+\mu) \nabla \psi & =f \text { in } \Omega, \\
\psi-\nabla . u & =0 \text { in } \Omega, \\
A u+B\left(\mu \frac{\partial u}{\partial n}+\lambda \nabla . u n\right) & =g \text { on } \Gamma .
\end{aligned}\right.
$$

For more information, the reader is invited to consult the article (MH. Sadd et al. 2005, [17], [16], [20], [21]).

The mathematical model of the Navier-lamé system with the generalized boundary condition noted $C_{A, B}$ such that $A$ is called the Dirichlet matrix, while $B$ is the Neumann matrix, $\alpha$ and $\beta$ are two strictly positive constants such as:

$$
\alpha u . u \leq u^{t} B^{-1} A u \leq \beta u . u, \forall u \in \mathbb{R}^{2} .
$$

|| . || defines a matrix norm. We assume :

- if $\||| A|| \ll \mid\| B \|$, puis $C_{A, B}$ est la condition aux limites de Neumann.

- if ||$|B||| \ll|||A|||$ puis $C_{A, B}$ est la condition aux limites de Dirichlet.

We need the following functional spaces:

$$
\begin{gathered}
h^{1}(\Omega)=\left\{u: \Omega \rightarrow \mathbb{R} \backslash u, \frac{\partial u}{\partial x}, \frac{\partial u}{\partial y} \in L^{2}(\Omega)\right\}, \\
V(\Omega)=H^{1}(\Omega)=\left[h^{1}(\Omega)\right]^{2}, \\
M(\Omega)=L_{0}^{2}(\Omega)=\left\{q \in L^{2}(\Omega) \backslash \int_{\Omega} q=0\right\} .
\end{gathered}
$$

The existence and the uniqueness of the weak formulation obtained is established in the papers [19], [18].

These spaces are less restrictive using the generalized boundary condition $C_{A, B}$.

We multiply the members of the equation (13) by the test function $v \in V(\Omega)$ for the displacement and $q \in M(\Omega)$ for the divergence of displacement, then we integrate and we apply Green's theorem and the generalized boundary condition $C_{A, B}$ we obtain the following variational formulation described as follows:

Find $(u, \psi) \in V(\Omega) \times M(\Omega)$ such as:

$$
\left\{\begin{array}{r}
\int_{\Omega} \mu \nabla u: \nabla v d \Omega+\int_{\Gamma} B^{-1} A u . v d \Gamma \\
-\int_{\Gamma} \mu \psi n \cdot v d \Gamma+\int_{\Omega}(\lambda+\mu) \psi \nabla \cdot v d \Omega \\
=\int_{\Omega} f \cdot v d \Omega+\int_{\Gamma} B^{-1} g . v d \Gamma \\
\int_{\Omega}(\lambda+\mu) q \nabla \cdot u d \Omega-\int_{\Omega}(\lambda+\mu) \psi q d \Omega=0 .
\end{array}\right.
$$


The weak formulation (18) is rewritten as follows: Find $(u, \psi) \in V(\Omega) \times M(\Omega)$ such as:

$$
\left\{\begin{array}{c}
a(u, v)+b_{\Gamma}(v, \psi)=L(v) \forall v \in V_{0}(\Omega), \\
b(u, q)-d(\psi, q)=0 \forall q \in M(\Omega) .
\end{array}\right.
$$

With the following bilinear forms:

$$
\left\{\begin{array}{r}
a(u, v)=\int_{\Omega} \mu \nabla u: \nabla v d \Omega+\int_{\Gamma} B^{-1} A u . v d \Gamma, \\
b(v, q)=\int_{\Omega}(\lambda+\mu) q \nabla \cdot v d \Omega, \\
b_{\Gamma}(v, q)=b(v, q)-\int_{\Gamma} \mu q n . v d \Gamma, \\
d(\psi, q)=\int_{\Omega}(\lambda+\mu) \psi q d \Omega, \\
L(v)=\int_{\Omega} f \cdot v d \Omega+\int_{\Gamma} B^{-1} g \cdot v d \Gamma .
\end{array}\right.
$$

According to (20), one notices that one resulted in a total insertion of the boundary conditions in the weak formulation of the problem, that allows us to make flat the numerical computations which one will make thereafter in part of the numerical resolution of the problem.

\section{MIXED FINITE ELEMENT APPROXIMATION WITH MINI-ELEMENT}

\section{A. Choice of a less restrictive approximate space}

In this section we will implement the mixed finite element method $(P 1+$ bubble,$P 1)$ to solve the Navier-Lamé equation with the generalized boundary condition $C_{A, B}$.

For this purpose, we indicate the privileges granted to us by this type of condition in the choice of the appropriate interpolation space which is less restrictive. This makes it easier for us to choose the basic functions suitable for our approximate problem.

In numerical analysis, the mixed finite element method also called hybrid finite element method is a finite element method in which additional independent variables are presented as nodal variables during the discretization of an equation problem. partial differential. Additional independent variables are limited by the use of Lagrange multipliers.

To distinguish the mixed finite element method from the classical finite element method is that the latter do not present such additional independent variables. They are also called irreducible finite element methods. The method of mixed finite elements is effective for certain problems badly formulated numerically by discretization by using the method of the irreducible finite elements, by way of example calculates fields of stress and deformation in an incompressible elastic body which was treated by C.olek et al. 2013 [11].

To apply the mixed finite element method $(P 1+$ bubble $/ P 1)$, we approach the problem by the standard Galerkin method.

For more explanations see the references (V. Girault et al. 1981. [5], [6], [3], [13], [7], [10], [9], [12].

Let $V_{h}$ the space of interpolation by displacement of finite element and $M_{h}$ is the space of interpolation by divdisplacement of finite element (corresponding to the spaces of the continuous problem respectively $V(\Omega)$ and $M=L_{0}^{2}(\Omega)$. The functions of the space $V_{h}$ are determined by their values in each of the vertices of the mesh. Besides, the dimension of the space $V_{h}$ is $N-n_{s}$, with $N$ being the global number of vertices and of $n_{s}$ the number of vertices at the limits. The mixed finite element problem is defined as follows:

We define approximate spaces in the following form: (for ease of formulation, we note the restriction of $u_{h}$ and $\psi_{h}$ on $K$ by $u_{h}$ and $\psi_{h}$ respectively) For everything, $\left(u_{h}, \psi_{h}\right) \in V_{h} \times M_{h} \subset$ $V \times M$

$$
\begin{array}{r}
u_{h}=\sum_{i=1}^{3} \alpha_{i}^{K} \varphi_{i}^{K}+\beta^{K} \mu^{K}(x), \alpha_{i}^{K}, \beta^{K} \in \mathbb{R}^{2} \\
\psi_{h}=\sum_{i=1}^{3} \theta_{i}^{K} \varphi_{i}^{K}, \theta_{i}^{K} \in \mathbb{R}, \forall K \in \mathrm{T}_{h} .
\end{array}
$$

While the less constraining approximate spaces $V_{h}$ and $M_{h}$ are written in the form:

$$
\begin{gathered}
V_{h}=\left\{u_{h} \in V /\left.u_{h}\right|_{K}=\sum_{i=1}^{3} \alpha_{i}^{K} \varphi_{i}^{K}+\beta^{K} \mu^{K}(x), \forall K \in T_{h}\right\}, \\
M_{h}=\left\{\psi_{h} \in M /\left.\psi_{h}\right|_{K}=\sum_{i=1}^{3} \theta_{i}^{K} \varphi_{i}^{K}, \forall K \in T_{h}\right\} .
\end{gathered}
$$

The spaces $V_{h}$ and $M_{h}$ are less restrictive thanks to the boundary conditions $C_{A, B}$, which exempts us to create the space of approximation of the Lagrange multiplier caused by the non-homogeneous Dirichlet boundary condition.

The approximate problem is formulated in the form: Find $\left(u_{h}, \psi_{h}\right) \in V_{h} \times M_{h}$

$$
\left\{\begin{array}{r}
a\left(u_{h}, v_{h}\right)+b_{\Gamma}\left(v_{h}, \psi_{h}\right)=L_{h}\left(v_{h}\right), \\
b\left(u_{h}, q_{h}\right)-d_{h}\left(\psi_{h}, q_{h}\right)=0 .
\end{array}\right.
$$

$\forall v_{h} \in V_{h}, \forall q_{h} \in M_{h}$, dont :

$$
\begin{gathered}
a\left(u_{h}, v_{h}\right)=\int_{K} \mu \nabla u_{h}: \nabla v_{h} d K \\
+\int_{\Gamma_{h}} B^{-1} A u_{h} \cdot v_{h} d \Gamma_{h}, \\
b\left(v_{h}, q_{h}\right)=\int_{K}(\lambda+\mu) q_{h} \nabla \cdot v_{h} d K, \\
b_{\Gamma}\left(v_{h}, q_{h}\right)=b\left(v_{h}, q_{h}\right)-\int_{\Gamma_{h}} \mu q_{h} n_{K} \cdot v_{h} d \Gamma_{h}, \\
d\left(\psi_{h}, q_{h}\right)=\int_{K}(\lambda+\mu) \psi_{h} q_{h} d K, \\
L\left(v_{h}\right)=\int_{K} f \cdot v_{h} d K+\int_{\Gamma_{h}} B^{-1} g_{h} \cdot v_{h} d \Gamma_{h} .
\end{gathered}
$$

$\Gamma_{h}=\Gamma \bigcap \partial K$ and $n_{K}$ normal over $K$. The existence of a single solution of the mixed formulation (25) is proved by the use of the continuity of the bilinear forms $a$ on $V_{h} \times V_{h}, b_{\Gamma}$ on $V_{h} \times M_{h}, b$ on $V_{h} \times M_{h}$ and $d$ on $M_{h} \times M_{h}$ which is clear using Korn's inequality. On the other hand, the coercivity of the bilinear form $a$ on $V_{h}$ and $\mathrm{d}$ on $M_{h}$ is held using their 
coercivity on $V(\Omega)$ and $M(\Omega)$ respectively from $V_{h} \subset V(\Omega)$. The uniform condition of inf - sup of the bilinear form $b$ and the bilinear form $b_{\Gamma}$ on $V_{h} \times M_{h}$ is treated by D.N Arnold et al. 1984 in [2] and O.koubaiti and .al 2018 in [19], [18].

\section{B. Exclusion of degrees of freedom associated with bubbles}

To eliminate the degrees of freedom associated with bubbles, it suffices to express $\beta^{K}$ in the relation (21) as a function of $f$, of the unknown $\psi$ and of the function bubble $\mu^{K}$.

Indeed, let $\left(i_{j}\right)_{j=1,2,3}$ be the (global) numbers of the 3 vertices of the triangle $K$. We have $\forall v_{h} \in V_{h}$

$$
a\left(u_{h}, v_{h}\right)=\int_{K} \mu \nabla u_{h}: \nabla v_{h} d K+\int_{\Gamma_{h}} B^{-1} A u_{h} . v_{h} d \Gamma_{h},
$$

In particular, if we take for $l=1.2, v_{h}^{l}=\mu^{K}$, since the bubbles are zero on the edge of $K$ then the equation (32) becomes:

$$
a\left(u_{h}^{l}, \mu^{K}\right)=\int_{K} \mu \nabla u_{h}^{l}: \nabla \mu^{K} d K,
$$

From (21) we have $\left.u_{h}^{l}\right|_{K}=\left(\sum_{j=1}^{3} \alpha_{i_{j}}^{l} \varphi_{i_{j}}^{K}\right)+\beta_{l}^{K} \mu^{K}(x)$

$$
\begin{aligned}
& a\left(u_{h}^{l}, \mu^{K}\right)=\int_{K} \mu \nabla\left[\left(\sum_{j=1}^{3} \alpha_{i_{j}}^{l} \varphi_{i_{j}}^{K}\right)+\beta_{l}^{K} \mu^{K}(x)\right] . \nabla \mu^{K} d K, \\
= & \int_{K} \mu \sum_{j=1}^{3} \alpha_{i_{j}}^{l} \nabla \varphi_{i_{j}}^{K} . \nabla \mu^{K} d K+\int_{K} \mu \beta_{l}^{K} \nabla \mu^{K}(x) \cdot \nabla \mu^{K} d K .
\end{aligned}
$$

By applying Green's formula we have :

$\int_{K} \nabla \varphi_{i_{j}}^{K} \cdot \nabla \mu^{K} d K=-\int_{K} \triangle \varphi_{i_{j}}^{K} \mu^{K} d+\int_{\partial K} \nabla \varphi_{i_{j}}^{K} \cdot n \mu^{K} d \partial K$

Since the $\varphi_{i_{j}}$ are affine, then $\Delta \varphi_{i_{j}}=0$ et $\mu^{K}=0$ on $\partial K$, we obtain :

$$
a\left(u_{h}^{l}, \mu^{K}\right)=\int_{K} \mu \beta_{l}^{K} \nabla \mu^{K}(x) \cdot \nabla \mu^{K} d K .
$$

According to the system (25) we have :

$$
a\left(u_{h}, \mu^{K}\right)=L\left(\mu^{K}\right)-b_{\Gamma}\left(\mu^{K}, \psi_{h}\right)
$$

Which means that for everything $l=1,2$ :

$\int_{K} \mu \beta_{l}^{K} \nabla \mu^{K} \cdot \nabla \mu^{K} d K=\int_{K} f^{l} \mu^{K} d K-\int_{K}(\lambda+\mu) \psi_{h} \frac{\partial \mu^{K}}{\partial x_{l}} d K$

Since $\left.\psi_{h}\right|_{K}=\sum_{j=1}^{3} \theta_{i_{j}} \varphi_{i_{j}}^{K}$, alors

$$
\beta_{l}^{K} \mu\left\|\nabla \mu^{K}\right\|_{0, K}^{2}=\int_{K} f^{l} \mu^{K} d K-(\lambda+\mu) \sum_{j=1}^{3} \theta_{i_{j}} \int_{K} \varphi_{i_{j}}^{K} \frac{\partial \mu^{K}}{\partial x_{l}} d K
$$

Finally, we get for all $l=1,2$ :

$\beta_{l}^{K}=\frac{1}{\mu\left\|\nabla \mu^{K}\right\|_{0, K}^{2}}\left(\int_{K} f^{l} \mu^{K} d K-(\lambda+\mu) \sum_{j=1}^{3} \theta_{i_{j}} \int_{K} \varphi_{i_{j}}^{K} \frac{\partial \mu^{K}}{\partial x_{l}} d K\right)$

\section{Algebric problem}

In this section, we present the matrices $A, B_{\gamma}, B, D$, $L$ linked to discrete bilinear forms $a_{h}, b_{\Gamma h}, b_{h}, d_{h}, L_{h}$ respectively and we express the bilinear forms according to the operators as well defined here :

$$
\left\{\begin{array}{r}
a_{h}\left(u_{h}, v_{h}\right)=\left(A u_{h}, v_{h}\right), \\
b_{\Gamma h}\left(v_{h}, q_{h}\right)=\left(B v_{h}, q_{h}\right), \\
b_{h}\left(u_{h}, q_{h}\right)=\left(B v_{h}, q_{h}\right), \\
d_{h}\left(\psi_{h}, q_{h}\right)=\left(D \psi_{h}, q_{h}\right), \\
L_{h}\left(v_{h}\right)=L v_{h},
\end{array}\right.
$$

$\forall\left(u_{h}, v_{h}\right) \in V_{h}(\Omega) \times V_{h}(\Omega)$,

$\forall\left(\psi_{h}, q_{h}\right) \in M_{h}(\Omega) \times M_{h}(\Omega)$

With (42), we find that the discrete formulation (25) can be expressed as a system of equations according to the following form :

$$
\left\{\begin{array}{r}
A u_{h}+B_{\Gamma}^{t} \psi_{h}=L \\
B u_{h}-D \psi_{h}=0
\end{array}\right.
$$

Then the discrete formulation can also be expressed by a system of linear equations as follows :

$$
\left(\begin{array}{cc}
A & B_{\Gamma}^{t} \\
B & -D
\end{array}\right)\left(\begin{array}{l}
u_{h} \\
\psi_{h}
\end{array}\right)=\left(\begin{array}{l}
L \\
0
\end{array}\right)
$$

With $u_{h}=\left(u_{x}, u_{y}\right)^{t}$, we can express the algebraic system (43) as follows :

$$
\left(\begin{array}{ccc}
A_{x} & 0 & B_{\Gamma, x}^{t} \\
0 & A_{y} & B_{\Gamma, y}^{t} \\
B_{x} & B_{y} & -D
\end{array}\right)\left(\begin{array}{c}
u_{x} \\
u_{y} \\
\psi
\end{array}\right)=\left(\begin{array}{c}
L_{x} \\
L_{y} \\
0
\end{array}\right)
$$

Let $\left\{\varphi_{1} ; \varphi_{2} \ldots ; \varphi_{n}\right\}$ the finite element base formed of scalar functions $\varphi_{i}, i=1 \ldots n$. In practice, the two components $\left(u_{h}^{x}, u_{h}^{y}\right)$ of $u_{h}$ are always appreciated by a finite element of space. Let $N$ be the number of nodes in the finite element mesh, and $n=N-n_{s}$ with $n s$ the number of vertices on the edges. The base of the space $V_{h}$ is:

$$
\begin{array}{r}
B_{V_{h}}=\left\{\phi_{1}=\left(\varphi_{1}, 0\right) \ldots \phi_{n}\right. \\
\left.=\left(\varphi_{n}, 0\right), \phi_{n+1}=\left(0, \varphi_{1}\right) \ldots \phi_{2 n}=\left(0, \varphi_{n}\right)\right\},
\end{array}
$$

Then, $u_{h}=\left(u_{h}^{x}, u_{h}^{y}\right) \in V_{h}$ can be given by the relation :

$$
u_{h}=u_{1}^{x} \phi_{1}+\ldots+u_{n}^{x} \phi_{n}+u_{1}^{y} \phi_{n+1}+\ldots+u_{n}^{y} \phi_{2 n},
$$

For a given triangle $K_{k}$, the displacement field $u_{h}$ and the divergence $\psi_{h}$ are approximated by linear combinations of the basic functions of the following form :

$$
\begin{array}{r}
u_{h}^{x}=\sum_{i=1}^{3} u_{k_{i}}^{x} \varphi_{k_{i}}+u_{x b} \varphi_{b}, \\
u_{h}^{y}=\sum_{i=1}^{3} u_{k_{i}}^{y} \varphi_{k_{i}}+u_{y b} \varphi_{b}, \\
\psi_{h}=\sum_{i=1}^{3} \psi_{k_{i}} \varphi_{k_{i}}
\end{array}
$$


We rewrite the system (25) on an element $K_{k}$ of triangulation. For all $k=1, \ldots n_{t}$, and in particular we take $v_{h}^{x}=v_{h}^{y}=$ $\varphi_{k_{j}}+\varphi_{b}$ and $q_{h}=\varphi_{k_{j}}$, for all $j=1,2,3$.

$$
\left\{\begin{array}{r}
a_{x}\left(u_{h}^{x}, \varphi_{k_{j}}+\varphi_{b}\right)+b_{\Gamma}^{x}\left(\varphi_{k_{j}}+\varphi_{b}, \psi_{h}\right)=L_{x}\left(\varphi_{k_{j}}+\varphi_{b}\right), \\
a_{y}\left(u_{h}^{y}, \varphi_{k_{j}}+\varphi_{b}\right)+b_{\Gamma}^{y}\left(\varphi_{k_{j}}+\varphi_{b}, \psi_{h}\right)=L_{y}\left(\varphi_{k_{j}}+\varphi_{b}\right), \\
b^{x}\left(u_{h}^{y}, \varphi_{k_{j}}\right)+b^{y}\left(u_{h}^{y}, \varphi_{k_{j}}\right)-d\left(\psi_{h}, \varphi_{k_{j}}\right)=0 .
\end{array}\right.
$$

But, we have :

$$
\begin{array}{r}
u_{h}^{x}=\sum_{i=1}^{3} u_{k_{i}}^{x} \varphi_{k_{i}}+u_{b}^{x} \varphi_{b}, \\
u_{h}^{y}=\sum_{i=1}^{3} u_{k_{i}}^{y} \varphi_{k_{i}}+u_{b}^{y} \varphi_{b}, \\
\psi_{h}=\sum_{i=1}^{3} \psi_{k_{i}} \varphi_{k_{i}} .
\end{array}
$$

The system (52) becomes :

$$
\left\{\begin{array}{r}
a_{x}\left(\sum_{i=1}^{3} u_{k_{i}}^{x} \varphi_{k_{i}}+u_{b}^{x} \varphi_{b}, \varphi_{k_{j}}+\varphi_{b}\right) \\
+b_{\Gamma}^{x}\left(\varphi_{k_{j}}+\varphi_{b}, \sum_{i=1}^{3} \psi_{k_{i}} \varphi_{k_{i}}\right)=L_{x}\left(\varphi_{k_{j}}+\varphi_{b}\right), \\
a_{y}\left(\sum_{i=1}^{3} u_{k_{i}}^{y} \varphi_{k_{i}}+u_{b}^{y} \varphi_{b}, \varphi_{k_{j}}+\varphi_{b}\right) \\
+b_{\Gamma}^{y}\left(\varphi_{k_{j}}+\varphi_{b}, \sum_{i=1}^{3} \psi_{k_{i}} \varphi_{k_{i}}\right)=L_{y}\left(\varphi_{k_{j}}+\varphi_{b}\right), \\
b^{x}\left(\sum_{i=1}^{3} u_{k_{i}}^{x} \varphi_{k_{i}}+u_{b}^{x} \varphi_{b}, \varphi_{k_{j}}\right)+b^{y}\left(\sum_{i=1}^{3} u_{k_{i}}^{y} \varphi_{k_{i}}+u_{b}^{y} \varphi_{b}, \varphi_{k_{j}}\right) \\
-d\left(\sum_{i=1}^{3} \psi_{k_{i}} \varphi_{k_{i}}, \varphi_{k_{j}}\right)=0 .
\end{array}\right.
$$

Since the bubble function $\varphi_{b}$ is zero on the edge of each element $K_{K}$ the system (56) becomes :

$$
\begin{aligned}
\int_{K_{k}}\left(\left(\sum_{i=1}^{3} u_{k_{i}}^{x} \mu \nabla \varphi_{k_{i}}\right)+u_{b}^{x} \nabla \varphi_{b}\right) \cdot\left(\nabla \varphi_{k_{j}}+\nabla \varphi_{b}\right) d K_{k} \\
+\int_{K_{k} \cap \Gamma_{h}}\left(B^{-1} A\right)_{11} \sum_{i=1}^{3} u_{k_{i}} \varphi_{k_{i}} \varphi_{k_{j}} d \Gamma_{h}+b_{\Gamma}\left(\varphi_{k_{j}}+\varphi_{b}, \sum_{i=1}^{3} \psi_{k_{i}} \varphi_{k_{i}}\right) \sum_{i=1}^{3} u_{k_{i}}^{y}\left(\int_{K_{k}} \mu \nabla \varphi_{k_{i}} \cdot \nabla \varphi_{k_{j}} d K_{k}+\int_{K_{k} \cap \Gamma_{h}} \alpha_{22} \varphi_{k_{i}} \varphi_{k_{j}} d \Gamma_{h}\right. \\
=L_{x}\left(\varphi_{k_{j}}+\varphi_{b}\right), \quad(57) \quad+u_{b}^{y} \int_{K_{k}} \nabla \varphi_{b} \cdot \nabla \varphi_{b} d K_{k}+b_{\Gamma}^{y}\left(\varphi_{k_{j}}+\varphi_{b}, \sum_{i=1}^{3} \psi_{k_{i}} \varphi_{k_{i}}\right)=L_{y}\left(\varphi_{k_{j}}+\varphi_{b}\right),
\end{aligned}
$$

$$
\begin{gathered}
\sum_{i=1}^{3} u_{k_{i}}^{y}\left(\int_{K_{k}} \mu \nabla \varphi_{k_{i}} \cdot \nabla \varphi_{k_{j}} d K_{k}+\int_{K_{k} \cap \Gamma_{h}}\left(B^{-1} A\right)_{22} \varphi_{k_{i}} \varphi_{k_{j}} d \Gamma_{h}\right) \\
+u_{b}^{y} \int_{K_{k}} \nabla \varphi_{b} . \nabla \varphi_{b} d K_{k}+b_{\Gamma}\left(\varphi_{k_{j}}+\varphi_{b}, \sum_{i=1}^{3} \psi_{k_{i}} \varphi_{k_{i}}\right)=L_{y}\left(\varphi_{k_{j}}+\varphi_{b}\right) \\
b^{x}\left(\left(\sum_{i=1}^{3} u_{k_{i}}^{x} \varphi_{k_{i}}\right)+u_{b}^{x} \varphi_{b}, \varphi_{k_{j}}\right)+b^{x}\left(\left(\sum_{i=1}^{3} u_{k_{i}}^{y} \varphi_{k_{i}}\right)+u_{b}^{y} \varphi_{b}, \varphi_{k_{j}}\right) \\
-d\left(\sum_{i=1}^{3} \psi_{k_{i}} \varphi_{k_{i}}, \varphi_{k_{j}}\right)=0
\end{gathered}
$$

We are going to express the equations (61), (62), (63) according to the two components of $u_{k_{i}}=\left(u_{k_{i}}^{x}, u_{k_{i}}^{y}\right)$, assuming that $\left(B^{-1} A\right)_{i j}=\alpha_{i j}$ and $\left(B^{-1}\right)_{i j}=\beta_{i j}$ for all $i, j=1,2$.

$$
\begin{gathered}
\sum_{i=1}^{3} u_{k_{i}}^{x}\left(\int_{K_{k}} \mu \nabla \varphi_{k_{i}} \cdot \nabla \varphi_{k_{j}} d K_{k}+\int_{K_{k} \cap \Gamma_{h}} \alpha_{11} \varphi_{k_{i}} \varphi_{k_{j}} d \Gamma_{h}\right. \\
+u_{b}^{x} \int_{K_{k}} \nabla \varphi_{b} \cdot \nabla \varphi_{b} d K_{k}+b_{\Gamma}^{x}\left(\varphi_{k_{j}}+\varphi_{b}, \sum_{i=1}^{3} \psi_{k_{i}} \varphi_{k_{i}}\right)=L_{x}\left(\varphi_{k_{j}}+\varphi_{b}\right),
\end{gathered}
$$

$$
\int_{K_{k}}\left(\left(\sum_{i=1}^{3} u_{k_{i}}^{y} \mu \nabla \varphi_{k_{i}}\right)+u_{b}^{y} \nabla \varphi_{b}\right) \cdot\left(\nabla \varphi_{k_{j}}+\nabla \varphi_{b}\right) d K_{k}
$$$$
+\int_{K_{k} \cap \Gamma_{h}}\left(B^{-1} A\right)_{22} \sum_{i=1}^{3} u_{k_{i}} \varphi_{k_{i}} \varphi_{k_{j}} d \Gamma_{h}+b_{\Gamma}\left(\varphi_{k_{j}}+\varphi_{b}, \sum_{i=1}^{3} \psi_{k_{i}} \varphi_{k_{i}}\right)
$$$$
b^{x}\left(\left(\sum_{i=1}^{3} u_{k_{i}}^{x} \varphi_{k_{i}}\right)+u_{b}^{x} \varphi_{b}, \varphi_{k_{j}}\right)+b^{y}\left(\left(\sum_{i=1}^{3} u_{k_{i}}^{y} \varphi_{k_{i}}\right)+u_{b}^{y} \varphi_{b}, \varphi_{k_{j}}\right)
$$$$
\sum_{i=1}^{3} u_{k_{i}}^{x}\left(\int_{K_{k}} \mu \nabla \varphi_{k_{i}} \cdot \nabla \varphi_{k_{j}} d K_{k}+\int_{K_{k} \cap \Gamma_{h}}\left(B^{-1} A\right)_{11} \varphi_{k_{i}} \varphi_{k_{j}} d \Gamma_{h}\right)
$$$$
+u_{b}^{x} \int_{K_{k}} \nabla \varphi_{b} . \nabla \varphi_{b} d K_{k}+b_{\Gamma}\left(\varphi_{k_{j}}+\varphi_{b}, \sum_{i=1}^{3} \psi_{k_{i}} \varphi_{k_{i}}\right)=L_{x}\left(\varphi_{k_{j}}+\varphi_{b}\right),
$$$$
b^{x}\left(\left(\sum_{i=1}^{3} u_{k_{i}}^{x} \varphi_{k_{i}}\right)+u_{b}^{x} \varphi_{b}, \varphi_{k_{j}}\right)+b^{y}\left(\left(\sum_{i=1}^{3} u_{k_{i}}^{y} \varphi_{k_{i}}\right)+u_{b}^{y} \varphi_{b}, \varphi_{k_{j}}\right)
$$$$
-d\left(\sum_{i=1}^{3} \psi_{k_{i}} \varphi_{k_{i}}, \varphi_{k_{j}}\right)=0
$$

$$
=L_{x}\left(\varphi_{k_{j}}+\varphi_{b}\right)
$$

$$
-d\left(\sum_{i=1}^{3} \psi_{k_{i}} \varphi_{k_{i}}, \varphi_{k_{j}}\right)=0
$$


The bilinear forms $b_{\Gamma}^{x}$ and $b_{\Gamma}^{y}$ appear in the equation (64), (65) are expressed in the following way :

$$
\begin{gathered}
b_{\Gamma}^{x}\left(\varphi_{k_{j}}+\varphi_{b}, \sum_{i=1}^{3} \psi_{k_{i}} \varphi_{k_{i}}\right)= \\
b_{\Gamma}^{x}\left(\varphi_{k_{j}}, \sum_{i=1}^{3} \psi_{k_{i}} \varphi_{k_{i}}\right)+b_{\Gamma}^{x}\left(\varphi_{b}, \sum_{i=1}^{3} \psi_{k_{i}} \varphi_{k_{i}}\right) \\
=\sum_{i=1}^{3} \psi_{k_{i}} b_{\Gamma}^{x}\left(\varphi_{k_{j}}, \varphi_{k_{i}}\right)+\sum_{i=1}^{3} \psi_{k_{i}} b_{\Gamma}^{x}\left(\varphi_{b}, \varphi_{k_{i}}\right) \\
=\sum_{i=1}^{3} \psi_{k_{i}}\left[\int_{K_{k}}(\mu+\lambda) \frac{\partial \varphi_{k_{j}}}{\partial x} \varphi_{k_{i}}-\int_{K_{k} \cap \Gamma_{h}} \mu \varphi_{k_{j}} n_{x} \varphi_{k_{i}}\right] \\
+\sum_{i=1}^{3} \psi_{k_{i}}\left[\int_{K_{k}}(\mu+\lambda) \frac{\partial \varphi_{b}}{\partial x} \varphi_{k_{i}}-\int_{K_{k} \cap \Gamma_{h}} \mu \varphi_{b} n_{x} \varphi_{k_{i}}\right] \\
=\sum_{i=1}^{3} \psi_{k_{i}}\left[\int_{K_{k}}(\mu+\lambda) \frac{\partial\left(\varphi_{k_{j}}+\varphi_{b}\right)}{\partial x} \varphi_{k_{i}}-\int_{K_{k} \cap \Gamma_{h}} \mu \varphi_{k_{j}} n_{x} \varphi_{k_{i}}\right]
\end{gathered}
$$

The bilinear form $d$ appears in the equation (66) is expressed in the following way :

$$
\begin{aligned}
d\left(\sum_{i=1}^{3} \psi_{k_{i}} \varphi_{k_{i}}, \varphi_{k_{j}}\right) & =\sum_{i=1}^{3} \psi_{k_{i}} d\left(\varphi_{k_{i}}, \varphi_{k_{j}}\right) \\
& =\sum_{i=1}^{3} \psi_{k_{i}} \int_{K_{k}} \varphi_{k_{i}} \varphi_{k_{j}}
\end{aligned}
$$

We inject the relationships (74), (71), (72), (69), (70) (67), (68) in the equations (64), (65) and (66). We obtain the following equations which will help us to extract all the elements from the local matrices associated with each element $K_{k}$ :For all $k=1,2 \ldots n_{t}$, and $j=1,2,3$

In the same way we will have :

$$
b_{\Gamma}^{y}\left(\varphi_{k_{j}}+\varphi_{b}, \sum_{i=1}^{3} \psi_{k_{i}} \varphi_{k_{i}}\right)=\sum_{i=1}^{3} \psi_{k_{i}}\left[\int_{K_{k}}(\mu+\lambda) \frac{\partial\left(\varphi_{k_{j}}+\varphi_{b}\right)}{\partial y} \varphi_{k_{i}}=\int_{K_{k}}\left(\varphi_{k_{j}}+\varphi_{b}\right) f_{x} d K_{k}+\int_{K_{k} \cap \Gamma_{h}}\left(\beta_{11}+\beta_{21}\right) g_{x} \varphi_{k_{j}} d \Gamma_{h}\right.
$$$$
\left.-\int_{K_{k} \cap \Gamma_{h}} \mu \varphi_{k_{j}} n_{y} \varphi_{k_{i}}\right]
$$

$$
\sum_{i=1}^{3} u_{k_{i}}^{x}\left(\int_{K_{k}} \mu \nabla \varphi_{k_{i}} \cdot \nabla \varphi_{k_{j}} d K_{k}+\int_{K_{k} \cap \Gamma_{h}} \alpha_{11} \varphi_{k_{i}} \varphi_{k_{j}} d \Gamma_{h}\right)
$$$$
+u_{b}^{x} \int_{K_{k}} \nabla \varphi_{b} . \nabla \varphi_{b} d K_{k}+\sum_{i=1}^{3} \psi_{k_{i}}\left[\int_{K_{k}}(\mu+\lambda) \frac{\partial\left(\varphi_{k_{j}}+\varphi_{b}\right)}{\partial x} \varphi_{k_{i}}\right.
$$

The linear forms $L_{x}$ and $L_{x}$ appear in the equations (64), (65) are expressed in the following way:

$$
\begin{aligned}
L_{x}\left(\varphi_{k_{j}}+\varphi_{b}\right)= & \int_{K_{k}}\left(\varphi_{k_{j}}+\varphi_{b}\right) f_{x} d K_{k} \\
& +\int_{K_{k} \cap \Gamma_{h}}\left(\beta_{11}+\beta_{21}\right) g_{x} \varphi_{k_{j}} d \Gamma_{h}, \\
L_{y}\left(\varphi_{k_{j}}+\varphi_{b}\right)= & \int_{K_{k}}\left(\varphi_{k_{j}}+\varphi_{b}\right) f_{y} d K_{k} \\
& +\int_{K_{k} \cap \Gamma_{h}}\left(\beta_{22}+\beta_{12}\right) g_{y} \varphi_{k_{j}} d \Gamma_{h} .
\end{aligned}
$$

Concerning the bilinear forms $b^{x}$ and $b^{y}$ appear in the system (66) are expressed in this way :

$$
\begin{gathered}
\sum_{i=1}^{3} u_{k_{i}}^{y}\left(\int_{K_{k}} \mu \nabla \varphi_{k_{i}} \cdot \nabla \varphi_{k_{j}} d K_{k}+\int_{K_{k} \cap \Gamma_{h}} \alpha_{22} \varphi_{k_{i}} \varphi_{k_{j}} d \Gamma_{h}\right) \\
+u_{b}^{y} \int_{K_{k}} \nabla \varphi_{b} . \nabla \varphi_{b} d K_{k}+\sum_{i=1}^{3} \psi_{k_{i}}\left[\int_{K_{k}}(\mu+\lambda) \frac{\partial\left(\varphi_{k_{j}}+\varphi_{b}\right)}{\partial y} \varphi_{k_{i}}\right. \\
\left.-\int_{K_{k} \cap \Gamma_{h}} \mu \varphi_{k_{j}} n_{y} \varphi_{k_{i}}\right] \\
=\int_{K_{k}}\left(\varphi_{k_{j}}+\varphi_{b}\right) f_{y} d K_{k}+\int_{K_{k} \cap \Gamma_{h}}\left(\beta_{22}+\beta_{12}\right) g_{y} \varphi_{k_{j}} d \Gamma_{h}
\end{gathered}
$$

$$
\begin{array}{r}
b^{x}\left(\left(\sum_{i=1}^{3} u_{k_{i}}^{x} \varphi_{k_{i}}\right)+u_{b}^{x} \varphi_{b}, \varphi_{k_{j}}\right)=b\left(\sum_{i=1}^{3} u_{k_{i}}^{x} \varphi_{k_{i}}, \varphi_{k_{j}}\right) \\
+b^{x}\left(u_{b}^{x} \varphi_{b}, \varphi_{k_{j}}\right) \\
=\sum_{i=1}^{3} u_{k_{i}}^{x} \int_{K_{k}} \frac{\partial \varphi_{k_{i}}}{\partial x} \varphi_{k_{j}}+u_{b}^{x} \int_{K_{k}} \frac{\partial \varphi_{b}}{\partial x} \varphi_{k_{j}} \\
b^{y}\left(\left(\sum_{i=1}^{3} u_{k_{i}}^{y} \varphi_{k_{i}}\right)+u_{b}^{y} \varphi_{b}, \varphi_{k_{j}}\right)=b\left(\sum_{i=1}^{3} u_{k_{i}}^{y} \varphi_{k_{i}}, \varphi_{k_{j}}\right) \\
+b^{y}\left(u_{b}^{y} \varphi_{b}, \varphi_{k_{j}}\right) \\
=\sum_{i=1}^{3} u_{k_{i}}^{y} \int_{K_{k}} \frac{\partial \varphi_{k_{i}}}{\partial y} \varphi_{k_{j}}+u_{b}^{y} \int_{K_{k}} \frac{\partial \varphi_{b}}{\partial y} \varphi_{k_{j}}
\end{array}
$$

$$
\begin{gathered}
\sum_{i=1}^{3} u_{k_{i}}^{x} \int_{K_{k}} \frac{\partial \varphi_{k_{i}}}{\partial x} \varphi_{k_{j}}+u_{b}^{x} \int_{K_{k}} \frac{\partial \varphi_{b}}{\partial x} \varphi_{k_{j}}+\sum_{i=1}^{3} u_{k_{i}}^{y} \int_{K_{k}} \frac{\partial \varphi_{k_{i}}}{\partial y} \varphi_{k_{j}} \\
\quad+u_{b}^{y} \int_{K_{k}} \frac{\partial \varphi_{b}}{\partial y} \varphi_{k_{j}}-\sum_{i=1}^{3} \psi_{k_{i}} \int_{K_{k}} \varphi_{k_{i}} \varphi_{k_{j}}=0
\end{gathered}
$$

The linear system (45), attached to the discrete system (43) is evaluated on each triangle $K_{k}$ for all $k=1, . . n_{t}$, with $n_{t}$ is the number of elements (triangles). The elements of the local matrices in miniscule and the global matrices are indicated by capital letters, and they are given by the following direct summation:

The elements of the local matrices on element $K_{k}$ for all $k=$ $1, \ldots n_{t}$ were designed starting from the equations (75), (76) 
and (77). They are given as :

$$
\begin{aligned}
& a_{j i}^{0}=\int_{K_{k}} \mu \nabla\left(\varphi_{k_{i}}+\varphi_{b}\right) \nabla\left(\varphi_{k_{j}}+\varphi_{b}\right) d K \\
& A_{0}=\sum_{K \in T_{h}} a_{j i}^{0}, \\
& a_{j i}^{x}=a_{i j}^{0}+\int_{E \cap K \subset \Gamma_{h}} \alpha_{11} \varphi_{i} \varphi_{j} d \Gamma_{h}, \\
& A_{x}=\sum_{K \in T_{h}} a_{j i}^{x}, \\
& a_{j i}^{y}=a_{i j}^{0}+\int_{E \cap K \subset \Gamma_{h}} \alpha_{22} \varphi_{i} \varphi_{j} d \Gamma_{h}, \\
& A_{y}=\sum_{K \in T_{h}} a_{j i}^{y}, \\
& b_{j i}^{x}=\int_{K}(\lambda+\mu) \frac{\partial\left(\varphi_{k_{i}}+\varphi_{b}\right)}{\partial x} \varphi_{k_{j}} d K \\
& B^{x}=\sum_{K \in T_{h}} b_{j i}^{x}, \\
& b_{j i}^{y}=\int_{K}(\lambda+\mu) \frac{\partial\left(\varphi_{k_{i}}+\varphi_{b}\right)}{\partial y} \varphi_{k_{j}} d K \\
& B^{y}=\sum_{K \in T_{h}} b_{j i}^{y}, \\
& b_{\Gamma_{j i}}^{x}=b_{j i}^{x}+\int_{E \cap K \subset \Gamma_{h}} \mu \varphi_{k_{i}} n_{i j} \varphi_{j} d E \\
& B_{\Gamma}^{x}=\sum_{K \in T_{h}} b_{\Gamma_{j i}}^{x}, \\
& b_{\Gamma_{j i}}^{y}=b_{i j}^{y}+\int_{E \cap K \subset \Gamma_{h}} \mu \varphi_{k_{i}} n_{i j} \varphi_{j} d E \\
& B_{\Gamma}^{y}=\sum_{K \in T_{h}} b_{\Gamma_{j i}}^{y}, \\
& d_{j i}=\int_{K}(\lambda+\mu) \varphi_{k_{i}} \varphi_{j} d K \\
& D=\sum_{K \in T_{h}} d_{j i}, \\
& l_{i}^{0 x}=\int_{K} f_{1}\left(\varphi_{k_{i}}+\varphi_{b}\right) d K \\
& l_{i}^{x}=l_{i}^{0 x}+\int_{E \cap K \subset \Gamma_{\Gamma h}}\left(\beta_{11}+\beta_{21}\right) g_{1} \varphi_{i} d E \\
& L^{x}=\sum_{K \in T_{h}} l_{i}^{x}, \\
& l_{i}^{0 y}=\int_{K} f_{2}\left(\varphi_{k_{i}}+\varphi_{b}\right) d K \\
& l_{i}^{y}=l_{i}^{0 y}+\int_{E \cap K \subset \Gamma_{\Gamma h}}\left(\beta_{12}+\beta_{22}\right) g_{2} \varphi_{i} \\
& L^{y}=\sum_{K \in T_{h}} l_{i}^{y},
\end{aligned}
$$

knowing that $f=\left(f_{1}, f_{2}\right)^{t}, g=\left(g_{1}, g_{2}\right)^{t}, n_{i j}=0,1$ or -1 .

\section{RSULTATS NUMRIQUES}

This section presents the numerical results obtained during the resolution of the Navier-Lamé problem in 2D. We present several numerical results obtained using the mixed finite element method $(P 1+$ bublle, $P 1)$. We compare these results with those of Abaqus software and of the ordinary finite element method. We consider a two-dimensional problem whose area

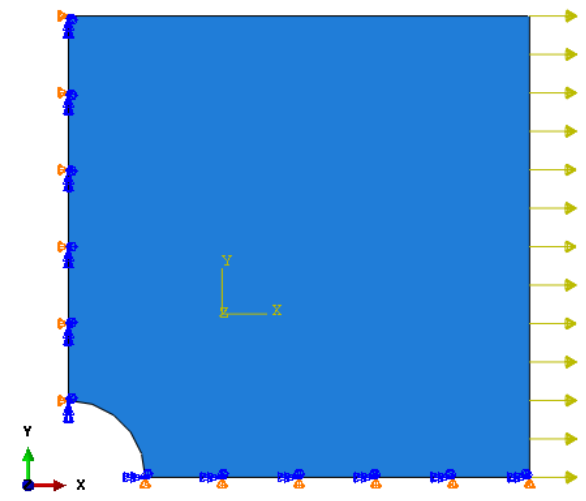

Fig. 1: A quarter of the plate with a hole in the center

of study $\Omega$ is a square plate of plexiglass material, with a hole in the center (see figure 1). The domain is homogeneous, for reasons of symmetry, we discretize that a quarter of the domain. We assume :

- $D=\left\{x^{2}+y^{2} \leq 1\right.$ such as $: 0 \leq x \leq 1$ et $\left.0 \leq y \leq 1\right\}$

- Le domaine $\Omega=[-5,5] \times[-5,5] \backslash D$

- $(E=2.90 G P a, \nu=0.4)$

We impose the following boundary conditions. On $\Gamma_{D}$, we take :

$$
A=\left(\begin{array}{ll}
1 & 0 \\
0 & 1
\end{array}\right), B=\left(\begin{array}{cc}
10^{-10} & 0 \\
0 & 10^{-10}
\end{array}\right)
$$

On $\Gamma_{N}$ :

$$
B=\left(\begin{array}{ll}
1 & 0 \\
0 & 1
\end{array}\right), A=\left(\begin{array}{cc}
10^{-10} & 0 \\
0 & 10^{-10}
\end{array}\right)
$$

- The domain $\Omega$ is stretched upwards $(x=5)$ with a surface charge $g=n=(1,0)$.

- Dirichlet conditions are: $u_{y}=0$ on: $[1,5] \times\{0\}, u_{x}=0$ on $\{0\} \times[1,5]$.

- The force loads take the value $f=(0,-(\mu+\lambda))^{t}$ for all the nodes.

- The rest of the border is free [8].

$n$ denotes the external normal on the edge $\partial \Omega$. We propose an exact solution: $u(x, y)=(x y, x y+x)$. This solution verifies the Navier-Lamé equation in the case where $f$ takes the value $f=(0,-(\mu+\lambda))^{t}$ on each node of the mesh.

The figures 2, 3, 4, 5 represent the displacements on the domain $\Omega$ defined above for the step $h=0.25$ for both MFEM methods with $C_{A, B}$ and that of Abaqus software.

Almost zero values of the displacements which appear at the edges $[1,5] \times\{0\}$ and $\{0\} \times[1,5]$. They thus reflect the boundary conditions of Dirichlet $u_{x}=0$ and $u_{y}=0$.

One observes then that displacements take maximum values in the vicinity of the edges of the condition of Neumann. 


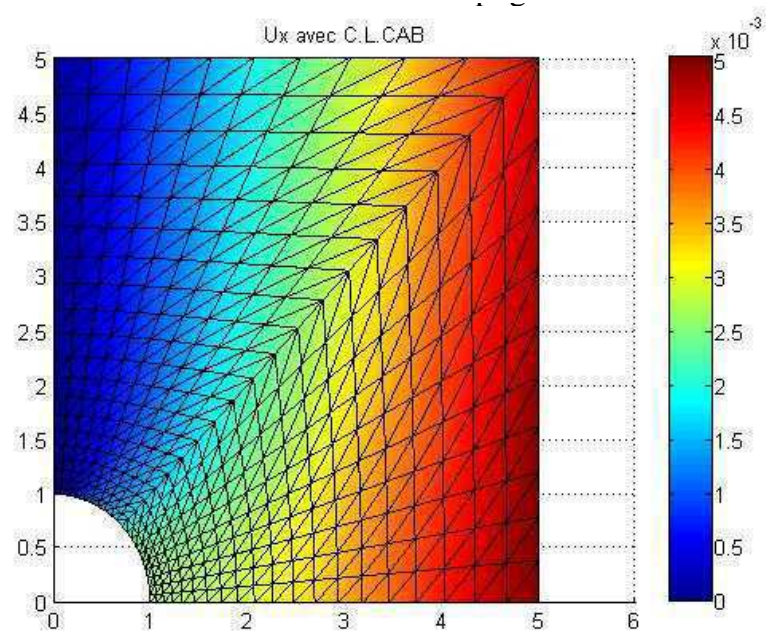

Fig. 2: displacement $u_{x}, h=0.25$, MFEM with $C_{A, B}$

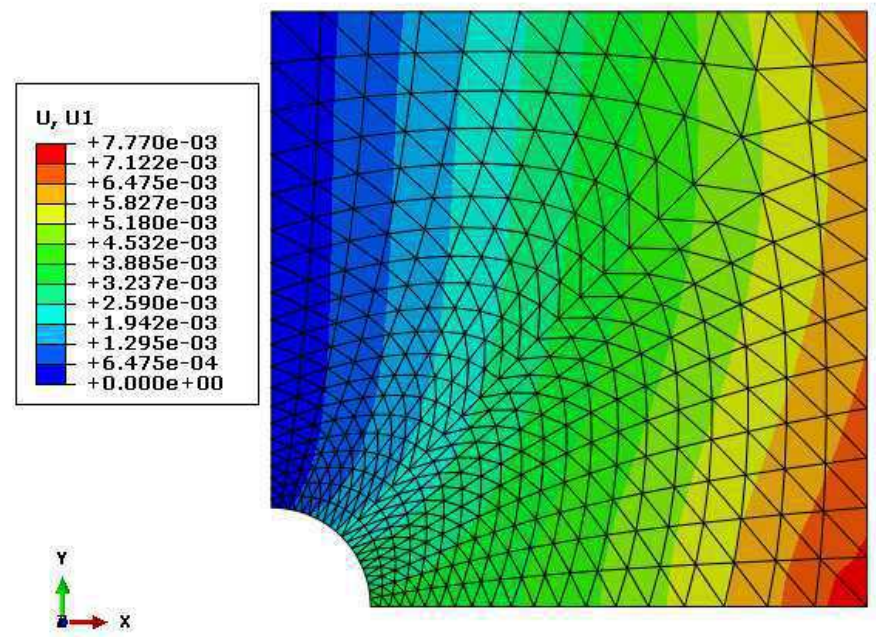

Fig. 3: displacement $u_{x}, h=0.25$, Abaqus

We notice that there is a similarity of results between our method MFEM with $C_{A, B}$ and those of the software Abaqus. We note that the results of Abaqus are based on the FEM method validated in the literature. This similarity of results means that the MFEM method is reliable and it provides a good solution. We calculate $\left\|u-u_{h}\right\|_{1, \Omega}$ for each of the two methods, then we get the two slopes using linear regression. The latter is presented in figures 10 and 11 for each method. They represent the linear correlation between $\log \left(\left\|u-u_{h}\right\|_{1, \Omega}\right)$ and $\log (h)$.

The errors $\left\|u-u_{h}\right\|_{1, \Omega}$ and $\left\|\psi-\psi_{h}\right\|_{0, \Omega}$ of the MFEM method with $C_{A, B}$ tends to 0 faster than those of the MFEM method with standards boundary conditions. This is reasonable. Indeed, with the generalized condition $C_{A, B}$ we reverse the matrix of the linear system directly without going through the algorithm of the iterative method which adds more error and more execution time. The figures 6, 7, 8, 9 represent the constraints $\sigma_{x x}$ and $\sigma_{y y}$ on the domain $\Omega$ defined above for the step $h=0.25$ for the two MFEM methods with $C_{A, B}$

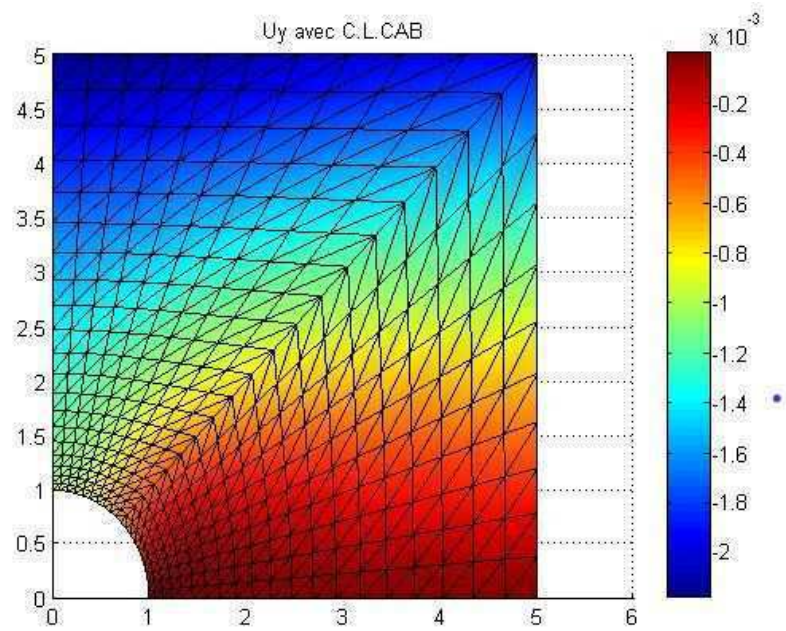

Fig. 4: displacements $u_{y}, h=0.25$, MFEM with $C_{A, B}$

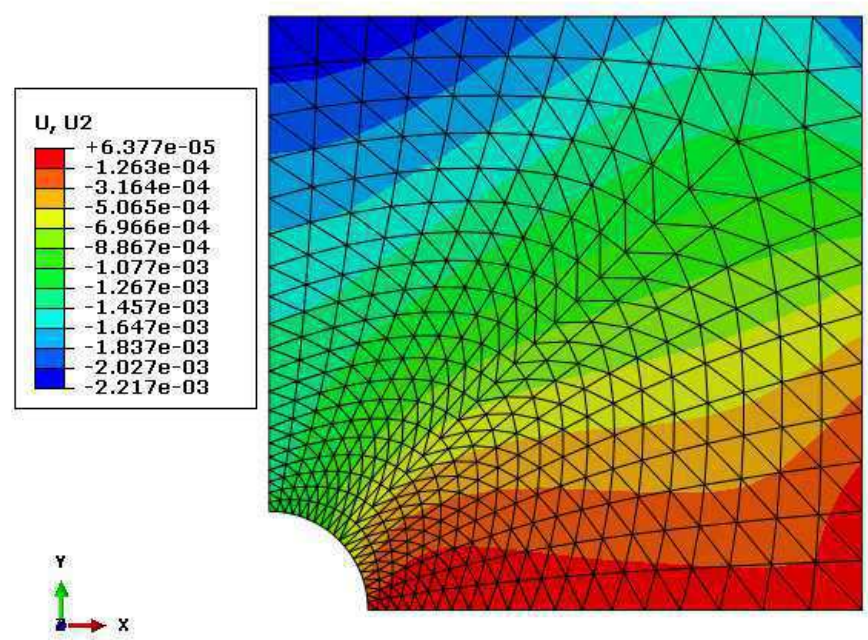

Fig. 5: displacements $u_{y}, h=0.25$, Abaqus

and that of Abaqus software.

We notice that the biggest constraints (over-stresses) are concentrated around the hole. The over-stresses are far from the traction field. So, the presence of a hole leads to a weakening of the structure due to over-stress around the hole. The table I

TABLE I: Errors table

\begin{tabular}{|c|c|c|c|}
\hline Number of knots $n p$ & 80 & 164 & 589 \\
\hline Number of elements $n t$ & 130 & 287 & 1099 \\
\hline Pas $\mathrm{h}$ & 0.3 & 0.2 & 0.1 \\
\hline$e_{\infty}$ MFEM with $C_{A, B}$ & 0.2252 & 0.2004 & 0.1699 \\
\hline$\left\|u-u_{h}\right\|_{1, \Omega}$ MFEM with $C_{A, B}$ & 0.0342 & 0.0298 & 0.0146 \\
\hline$\left\|\psi-\psi_{h}\right\|_{0, \Omega}$ MFEM with $C_{A, B}$ & 0.0567 & 0.0346 & 0.0264 \\
\hline$\left\|u-u_{h}\right\|_{1, \Omega}$ MFEM with standards B.C & 0.1360 & 0.1193 & 0.1074 \\
\hline$\left\|\psi-\psi_{h}\right\|_{0, \Omega}$ MFEM with standards B.C & 0.2032 & 0.1094 & 0.0894 \\
\hline$\left\|u-u_{h}\right\|_{1, \Omega}$ with FEM & 0.4924 & 0.2075 & 0.1448 \\
\hline
\end{tabular}

summarizes all the calculated errors. The error $e_{\infty}$ approaches zero when $h$ is small enough. Then the approximate solution $\psi_{h}$ obtained converges towards the discrete divergence (the value of the divergence of the displacement $u$ on each node). 


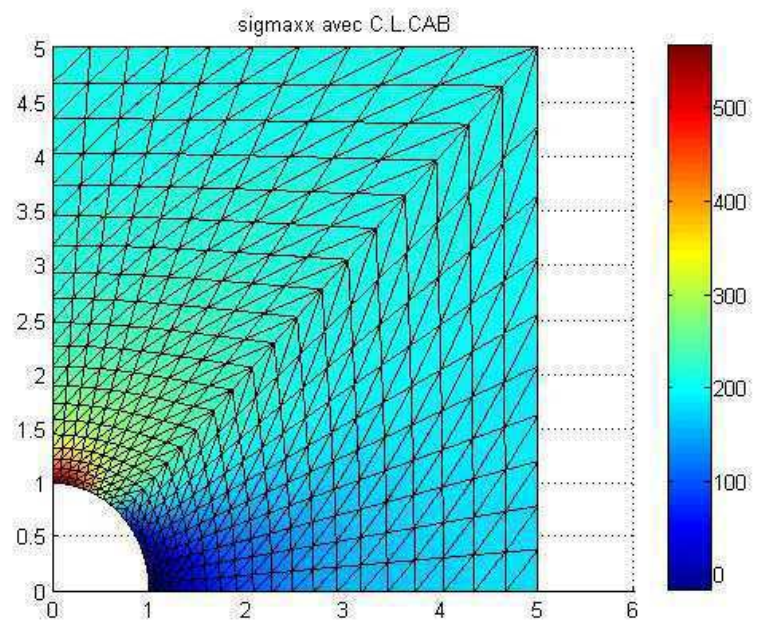

Fig. 6: Constraint $\sigma_{x x}, h=0.25$, MFEM with $C_{A, B}$
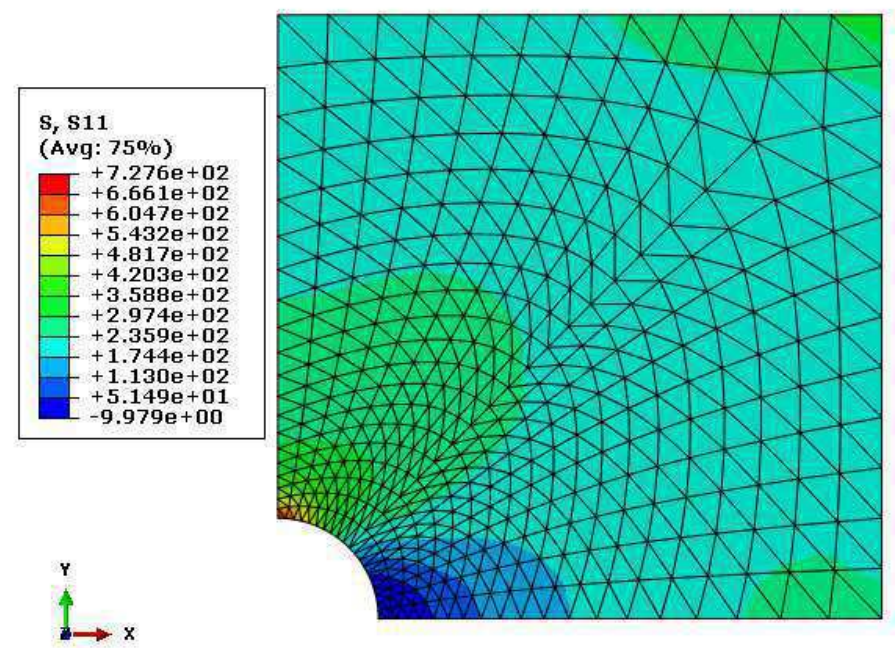

Fig. 7: Constraints $\sigma_{x x}, h=0.25$, Abaqus

Another objective of this numerical part is to test the stability of the divergence of the field of displacement of the numerical solution $u_{h}$. Therefore, we will calculate the error $e_{\infty}=\max _{i, j}\left(\left|\operatorname{divu}\left(x_{i}, y_{j}\right)-\psi_{i, j}\right|\right)$ for three meshes, and we observe the variation of this error according to the size of the mesh for $h \rightarrow 0$. This example shows that the mixed finite element method $(P 1+$ bubble, $P 1)$ is more effective than the ordinary method. This method makes it possible to calculate displacements and their divergences simultaneously. It guarantees the stability of these divergences on each node.

\section{Conclusion}

In this study, we proposed the mixed finite element method $(P 1+$ bubble, $P 1)$ for the resolution of the Navier-Lamé system. This problem is solved by the choice of generalized boundary conditions $C_{A, B}$ which makes the spaces of approximations less restrictive. As explained before, for the problem of elasticity, in which the form $a$ is coercive,the stability can always be obtained by an adequate enrichment

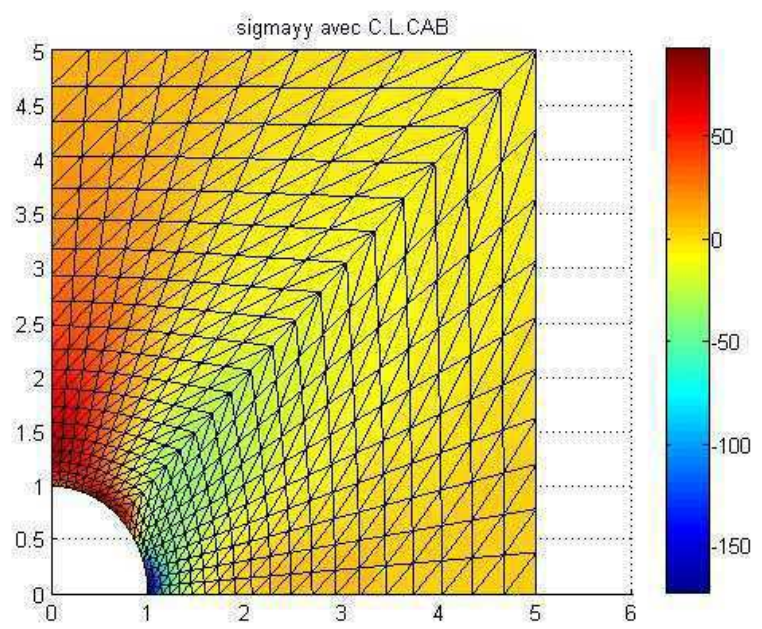

Fig. 8: Constraints $\sigma_{y y}, h=0.25$, MFEM with $C_{A, B}$

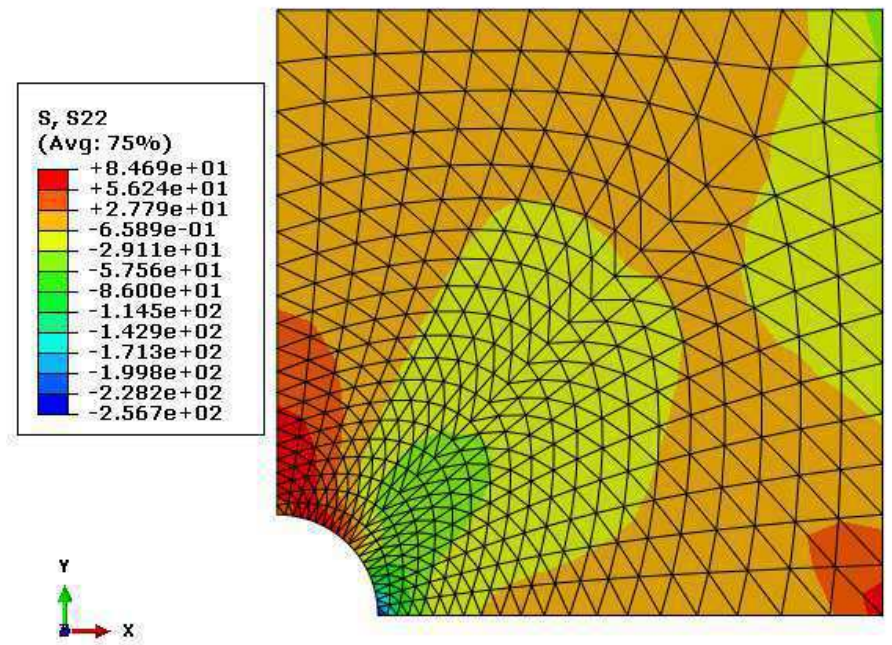

Fig. 9: Constraints $\sigma_{x x}, h=0.25$, Abaqus

of the displacement space. There are several ways to enrich the space. Take our case as an example, the torque element is unstable (linear displacement, linear divergence), and it can be stabilized by adding a single degree of freedom of internal displacement from a bubble function (see DN Arnold et al. 1984 [2]).

From the numerical results, we note that with the calculation of the slopes for each method, the slope obtained by the method $(P 1+b u b b l e, P 1)$ is more higher than the slope obtained by the classical method. This result means that the numerical solution $u_{a p p}$ obtained by the mixed finite element method $(P 1+$ bubble,$P 1)$ converges very quickly to the exact solution compared to the solution obtained by the classical method. The advantage of this problem with the boundary condition $C_{A, B}$ is at the programming level by Matlab. Just create a single code and then apply it to ordinary problems like Dirichlet and Neumann.

Also, one avoids the problem of constraints in the functional spaces, this fact facilitates numerical calculations. 


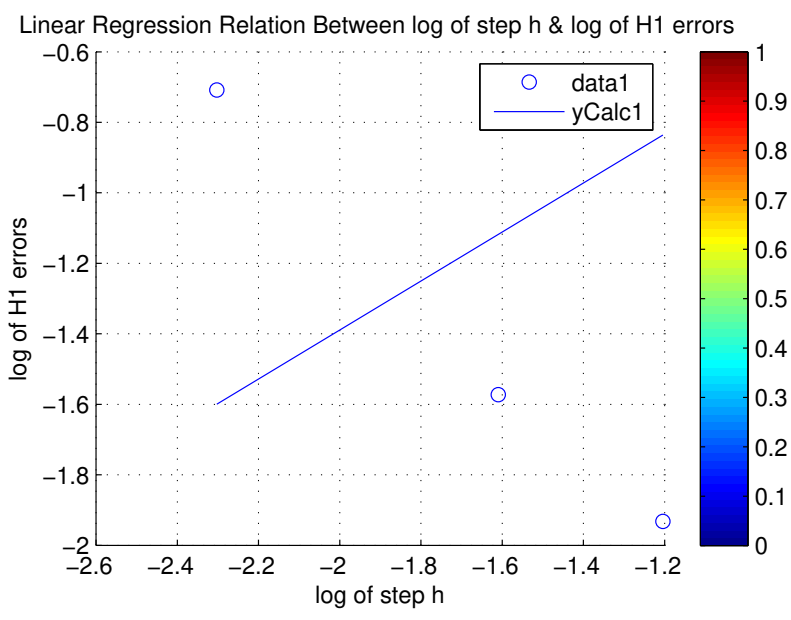

Fig. 10: La pente $\alpha=0.694$ with MFEM S.B.C Linear Regression Relation Between log of step h \& log of $\mathrm{H} 1$ errors

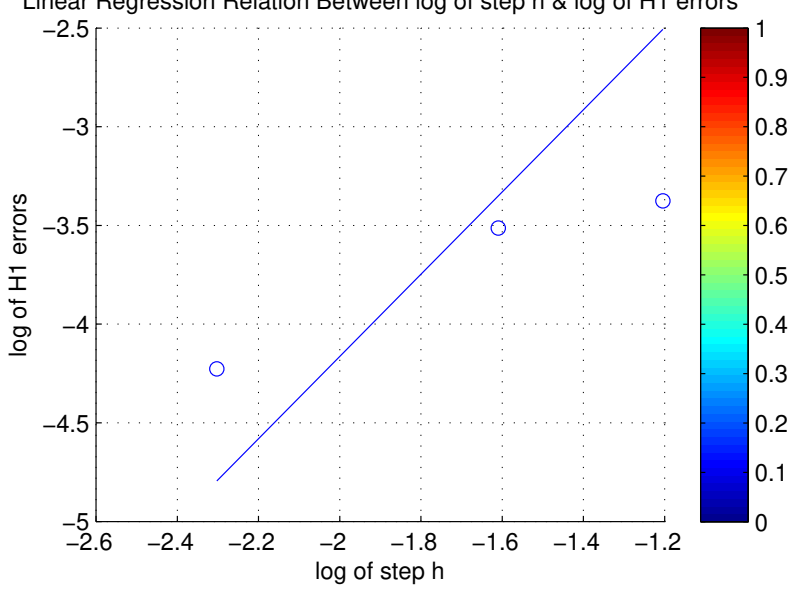

Fig. 11: La pente $\alpha=2.082$ with MFEM $C_{A, B}$

Finally, we have shown that solving the elasticity problem with boundary conditions $C_{A, B}$, using the element $(P 1+$ bubble, $P 1)$ is much more efficient than a standard implementation with ordinary finite elements.

\section{REFERENCES}

[1] J. Alberty, Kiel, C. Carstensen, Vienna, S. A. Funken, Kiel and R. Klose, Kiel. Matlab Implementation of the Finite Element Method in Elasticity. Computing. Springer-Verlag .Volume 69, Issue 3, pp 239-263. 2002.

[2] D.N. Arnold, F.Brezzi, M.Fortin. A stable finite element for the stokes equations. Estratto da Calcolo. vol: 21. 337 - 344. 1984.

[3] F.Brezzi and M.Fortin, Mixed and Hybrid Element Methods. SpringerVerlag. New York. 1991.

[4] Jonas Koko Limos. Vectorized Matlab Codes for the Stokes Problem with $(P 1+$ Bubble,$P 1)$ Finite Element. Universite Blaise Pascal -CNRS UMR 6158 ISIMA, Campus des Cezeaux - BP 10125, 63173 Aubire cedex, France. 2012.

[5] V.Girault and P. A. Raviart. Finite Element Approximation of the NavierStokes Equations, Springer-Verlag. Berlin Heiderlberg New York. 1981.

[6] Alexandre Ern. Aide-memoire Elements Finis, Dunod. Paris. 2005.

[7] D.Yang. Iterative schemes for mixed finite element methods with applications to elasticity and compressible flow problems. Numer. Math. vol: 93. pp.177-200. 2002.

[8] A. Geilenkothen. Constraint preconditioning for linear systems in elasticity. Proceedings in Applied Mathematics. pp: 481 - 482. 2003.

[9] Daniele Boffi, Franco Brezzi, Michel Fortin. Mixed Finite Element Methods and Applications, Springer, Berlin, Heidelberg. 2013.

[10] Gabriel N. Gatica. A Simple Introduction to the Mixed Finite Element Method. Springer. Theory and Applications. 2014.

[11] Olek C Zienkiewicz, Robert L Taylor and J.Z. Zhu. The Finite Element Method: Its basis and Fundamentals, Elsevier, Page Count: 756. 2013.

[12] Junichi Mtsumoto. A relationship between stabilized FEM and Bubble fonction element stabilization method with orthogonal basis for incompressible flows. Journal of applied mechanics. Vol 8. August. 2005.

[13] R.B.Kellogg and B. Liu, A finite element method for compressible Stokes equations,SIAM. J.Numer.Anal.,33 , pp. 780-788. 1996.

[14] R. A. Nicolaides. Existence, uniqueness and approximation for generalized saddle point problems, SIAM J. Numer. Anal., 19 , pp. 349 - 357. 1982.

[15] MH. Sadd. Elasticity: Theory, Application and Numerics. Amsterdam: Elsevier Butterworth Heinemann. 2005.

[16] SP. Timoshenko, Goodier JN. Theory of Elasticity. McGraw-Hill; New York: 1985.

[17] Sadd MH. Elasticity: Theory, Application and Numerics. Amsterdam: Elsevier Butterworth Heinemann; 2005.

[18] Ouadie Koubaiti, Jaouad El-mekkaoui, and Ahmed Elkhalfi. Complete study for solving Navier-Lamé equation with new boundary condition using mini element method. International journal of mechanics, Vol: 12, Pages: 46-58. 2018.

[19] Ouadie Koubaiti, Jaouad El-mekkaoui, and Ahmed Elkhalfi. Elasticity with mixed finite element. Communications in Applied Analysis. vol: 22. No: 4. 2018.

[20] Daniele Baraldi. Josan. An effective Galerkin Boundary Element Method for a 3D half-space subjected to surface loads . WSEAS Transactions on Applied and Theorical Mechanics. Volume 5, 2020.

[21] Sergy O. Glakov. On the Question of Nonlinear Fluctuations of Heavy Ropes WSEAS Transactions on Applied and Theorical Mechanics. Volume 15, 2020. 